\title{
The influence of age on atrial fibrillation recurrence after the maze procedure in patients with giant left atrium
}

\author{
Seung Hyun Lee, MD, Joon Bum Kim, MD, Won Chul Cho, MD, Cheol Hyun Chung, MD, \\ Sung Ho Jung, MD, Suk Jung Choo, MD, and Jae Won Lee, MD
}

\begin{abstract}
Objective: The presence of a giant left atrium (LA) is strongly associated with the recurrence of atrial fibrillation (AF) after the maze procedure. Therefore, we investigated the factors affecting the rhythm outcomes after the maze procedure in patients with a giant LA and AF-associated mitral valve disease to determine the best candidates for the maze procedure in this population.
\end{abstract}

\begin{abstract}
Methods: From January 1999 to December 2006, 224 patients with a giant LA ( $>60 \mathrm{~mm}$ ) underwent bi-atrial maze procedures combined with mitral valve surgery. Multivariate Cox regression analysis was performed to determine the risk factors for maze failure.
\end{abstract}

\begin{abstract}
Results: Follow-up was complete in all patients at a mean of $48.3 \pm 28.0$ months, during which 4 early and 9 late deaths occurred. Of the 218 early survivors, 24 had late AF recurrence. The 5-year AF-free rate was $87.1 \% \pm$ $4.3 \%$ in patients 50 years or younger and $77.3 \% \pm 4.1 \%$ in patients older than 50 years $(P=.001)$. Univariate analysis showed that advanced patient age ( $>50$ years), a longer duration of $\mathrm{AF}$, a fine AF pattern (wave length $<1 \mathrm{~mm}$ ), and increased cardiothoracic ratio $(>60 \%)$ were associated with late AF recurrence. On multivariate analysis, advanced patient age was the only significant, independent risk factor for AF recurrence.

Conclusions: Although giant LA is known to be a predictor of poor outcomes after the maze procedure, younger patients had more favorable rhythm outcomes than older patients, as shown by the superior freedom from AF recurrence. A more aggressive surgical approach in younger patients might eliminate AF, even in the presence of a giant LA. (J Thorac Cardiovasc Surg 2011;141:1015-9)
\end{abstract}

Because of its efficacy, the Cox maze procedure, first introduced in 1987, has become the standard method used to treat atrial fibrillation (AF). ${ }^{1}$ This procedure has been modified for easier and more effective interruption of micro-reentry circuits, resulting in normal sinus conversion rates as great as $90 \%{ }^{2}$ The risk factors for early or late recurrence after the modified maze procedure include $\mathrm{AF}$ duration $(>5$ years), low amplitude $(<1 \mathrm{~mm}) \mathrm{AF}$, advanced age, a greater cardiothoracic ratio $(>60 \%)$, and a giant $(>60 \mathrm{~mm})$ left atrium (LA). The LA size is a particularly important risk factor for failure of the maze procedure. ${ }^{3,4}$ A giant LA can be understood as a marker of arrhythmogenecity, in that pathologic changes such as atrial interstitial fibrosis, which is not involved in contraction, can easily induce $\mathrm{AF}^{5}$

Differences in the rates of sinus conversion, however, have been observed between younger and older patient groups in the presence of a giant LA, suggesting that youn-

\footnotetext{
From the Department of Thoracic and Cardiovascular Surgery, Asan Medical Center, University of Ulsan College of Medicine, Seoul, Korea.

Disclosures: Authors have nothing to disclose with regard to commercial support.

Received for publication March 23, 2010; revisions received July 12, 2010; accepted for publication Aug 2, 2010; available ahead of print Oct 11, 2010.

Address for reprints: Jae Won Lee, MD, Department of Thoracic and Cardiovascular Surgery, Asan Medical Center, University of Ulsan College of Medicine, 388-1

Pungnap-dong Songpa-gu, Seoul 138-736 South Korea (E-mail: jwlee@amc. seoul.kr).

$0022-5223 / \$ 36.00$

Copyright $(2011$ by The American Association for Thoracic Surgery

doi:10.1016/j.jtcvs.2010.08.036
}

ger patients with a giant LA might be better candidates for successful maze procedures, with an age cutoff for success. We, therefore, investigated the factors affecting rhythm outcomes after the maze procedure in patients with a giant LA and AF-associated mitral valve disease to determine the best candidates for the maze procedure in this population.

\section{MATERIALS AND METHODS}

From January 1999 to December 2006, 224 patients with a preoperative LA size larger than $60 \mathrm{~mm}$ underwent the bi-atrial maze procedure combined with mitral valve surgery. The data were collected prospectively. The preoperative factors included patient age, sex, preoperative ejection fraction, AF duration, AF pattern (coarse or fine), AF type (paroxysmal, persistent, or long-standing persistent), and cardiothoracic ratio. The intraoperative factors included abrasion energy source (microwave or cryocatheter), concomitant surgery, and mitral valve operation type. Postoperatively, the in-patient rhythms were monitored daily using standard 12-channel surface electrocardiography. The patients were routinely evaluated with 24-hour Holter monitoring before hospital discharge Follow-up electrocardiograms were performed at 3- to 6-month intervals during first 2 years and every year thereafter. Any symptoms suspicious of arrhythmia were assessed using 24-hour Holter monitoring during follow-up.

Our institution's ethical committee/institutional review board approved the present study.

\section{Definitions}

Recurrences or events of AF during the initial postablation blanking period of 3 months were defined as "early events." A failure of the maze procedure was defined as any recurrent $\mathrm{AF}$, atrial tachycardia, or atrial flutter at 


\section{Abbreviations and Acronyms}

$\mathrm{AF}=$ atrial fibrillation

LA $\quad=$ left atrium

least 3 months after stopping antiarrhythmic agents beyond the initial blanking period (late $\mathrm{AF}$ ).

A fine $\mathrm{AF}$ wave on the preoperative electrocardiogram was defined as a fibrillatory wave voltage in the $\mathrm{V}_{1}$ lead of less than $0.1 \mathrm{mV}$. The LA size was measured preoperatively using transthoracic echocardiography. Persistent AF was defined as AF that did not self-terminate, and paroxysmal $\mathrm{AF}$ was defined as intermittent or recurrent $\mathrm{AF}$.

\section{Surgical Technique of Maze Procedure}

The maze procedure was performed using a modified Cox-maze III technique, the details of which have been previously described. ${ }^{6}$ The resection of the right atrium auricle and the incision from the auricle to the tricuspid valve were abbreviated, and the cut and sewn lesions in the right atrium were replaced by ablation lines, except for the long oblique incision used to open the right atrium. Three ablation lines were made in the right atrium, on the intercaval, cavotricuspid, and free walls. LA lesions were treated by inferior extension of the left atriotomy to the orifice of the left inferior pulmonary vein, and four ablation lines were made, including inferior and superior ablation lines for pulmonary vein isolation, endocardial LA isthmus ablation, and epicardial coronary sinus ablation. The LA size was reduced in all patients by resection of the redundant posterior wall parallel to the mitral annulus. The degree of LA wall resection was determined by the preoperative LA size, with the target postoperative LA size of less than $50 \mathrm{~mm}$.

\section{Statistical Analysis}

Categorical variables are presented as frequencies and percentages, and continuous variables are expressed as the mean \pm standard deviation. Kaplan-Meier curves were used to delineate the time-related recurrence of AF. Stratified survival curves (freedom from AF) were plotted to explore unadjusted differences for variables of interest (log-rank test). For multivariate analysis, the Cox proportional hazards model was fitted with the interval to AF recurrence. Variables with $P \leq .20$ on the univariate analyses were candidates for the multivariate models. The results are expressed as the relative risk, with $95 \%$ confidence intervals. The predictive value of age for $\mathrm{AF}$ recurrence was evaluated using the area under the receiver operating characteristic curves, with the $95 \%$ confidence intervals. The optimal cutoff corresponded to the value with the greatest accuracy.

All reported $P$ values are 2 -sided and $P$ values of less than .05 were considered to indicate statistical significance. Statistical analyses were performed with the Statistical Package for Social Sciences, version 14.0, for Windows software (SPSS Inc, Chicago, Ill).

\section{RESULTS}

\section{Preoperative Patient Profiles (Table 1)}

Of the 224 patients, $97(43.3 \%)$ were men and 127 $(56.7 \%)$ were women. Their average age was $49.6 \pm 13.2$ years. The mean duration of AF was $6.4 \pm 7.0$ years, and the mean LA size was $67.6 \pm 7.6 \mathrm{~mm}$. Of the 224 patients, $103(46.0 \%)$ had fine AF, $24(10.7 \%)$ had paroxysmal AF, $52(23.2 \%)$ had persistent AF, and $158(70.5 \%)$ had long-standing AF. In addition, 62 patients $(27.7 \%)$ had rheumatic disease, $11(4.9 \%)$ had undergone previous cardiac surgery, and $44(19.6 \%)$ had a low ejection fraction
TABLE 1. Preoperative patient characteristics

\begin{tabular}{lc}
\hline Patients (n) & 224 \\
Age (y) & $49.6 \pm 13.2$ \\
Sex (n) & \\
Male & 97 \\
Female & 127 \\
Diabetes mellitus (n) & $9(4.0)$ \\
Hypertension (n) & $13(5.8)$ \\
Cardiothoracic ratio (\%) & $61.5 \pm 8.7$ \\
Redo-operation (n) & $11(4.9)$ \\
AF profile & \\
AF duration (y) & \\
$\quad$ Median & 4.0 \\
$\quad$ Range & $0-40$ \\
Coarse AF & 117 \\
Fine AF & 107 \\
AF type & \\
$\quad$ Paroxysmal & 19 \\
$\quad$ Persistent & 51 \\
$\quad$ Longstanding persistent AF (> 1 y) & 154 \\
Cause of mitral disease & \\
Degenerative & $62(27.7)$ \\
Rheumatic & $162(72.3)$ \\
Left ventricular ejection fraction $(\%)$ & $55.8 \pm 9.2$ \\
Left atrial dimension (mm) & $67.6 \pm 7.6$ \\
Surgical period (n) & \\
1999-2003 & $117(52.2)$ \\
2003-2006 & $107(47.8)$ \\
\hline Dain &
\end{tabular}

Data in parentheses are percentages. $A F$, Atrial fibrillation.

$(<50 \%)$. The mean preoperative cardiothoracic ratio was $61.5 \% \pm 8.7 \%$.

\section{Intraoperative Profiles and Operative Outcomes}

The mean total cardiopulmonary bypass time was $165.1 \pm 45.3$ minutes, and the mean total aortic crossclamp time was $117.8 \pm 30.1$ minutes. Of the 224 patients, 169 (75.4\%) underwent cryoablation and $55(24.6 \%)$ microwave ablation. Also, 118 patients $(52.7 \%)$ underwent mitral valve repair and $106(47.3 \%)$ mitral valve replacement. Concomitant operations included aortic valve surgery $(\mathrm{n}=192,85.7 \%)$ and coronary bypass surgery $(\mathrm{n}=32$, $14.3 \%)$.

Follow-up was complete for all patients, with a mean follow-up duration of $48.3 \pm 28.0$ months. During followup, 4 early and 9 late deaths occurred. Of the 218 early survivors, 24 had late $\mathrm{AF}$ recurrences. At the last rhythm follow-up examination, normal sinus rhythm conversion was observed in 172 patients $(76.8 \%)$.

\section{Risk Factor Analysis for Maze Failure and AF-Free Rate}

Of the 218 early survivors, 24 had a late AF recurrence. The 5-year AF-free rate was $87.1 \% \pm 4.3 \%$ for patients 50 years old or younger and $77.3 \% \pm 4.1 \%$ for those older than 50 years $(P=.001)$ (Figure 1). Univariate analysis 


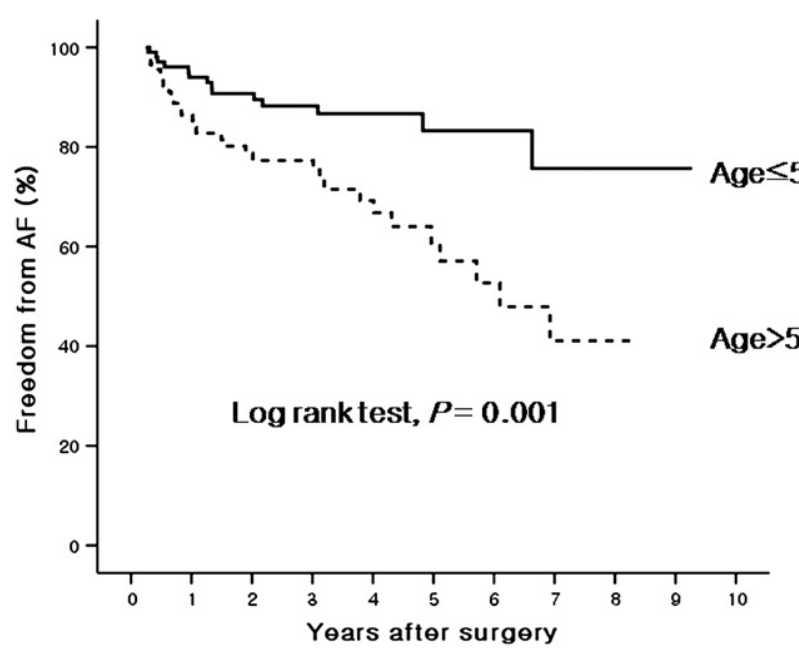

FIGURE 1. Kaplan-Meier analysis of atrial fibrillation $(A F)$ recurrence over time in patients with giant left atrium 50 years old or younger and older than 50 years.

showed that advanced patient age ( $>50$ years), longer AF duration, a fine AF pattern (wave length $<1 \mathrm{~mm}$ ), and increased cardiothoracic ratio $(>60 \%)$ were associated with late AF recurrence (Table 2). On multivariate analysis, however, advanced patient age was the only significant, independent factor for $\mathrm{AF}$ recurrence. When the predictive value of age for late $\mathrm{AF}$ was assessed using the receiver operating characteristic curve, the curve yielded an area under the curve of 0.67 (95\% confidence interval $0.58-0.75$; $P=.001)$. The greatest accuracy for the prediction of late AF was obtained at the cutoff value of 47.5 years, with $84.1 \%$ sensitivity and $54 \%$ specificity (Figure 2 ).

\section{DISCUSSION}

Although a giant LA has been considered a high risk factor for the maze procedure, ${ }^{7-9}$ recent findings have indicated that LA size was not critical for conversion to sinus rhythm using aggressive atrial reduction plasty. ${ }^{10} \mathrm{We}$ have routinely resected the left atrial posterior wall for size reduction, as well as using an alternative ablation line.

Although standardized procedures have been used in patients with $\mathrm{AF}$, differences in the results were observed among the groups of patients with a giant LA. Because some of these patients had 2 or more risk factors (eg, cardiothoracic ratio $>60 \%$, fine $\mathrm{AF}$ ), we hypothesized that a key factor might exist for successful conversion to normal sinus rhythm in patients with giant LA. Therefore, we analyzed the preoperative and intraoperative variables to determine whether any correlated with normal sinus rhythm conversion. In agreement with previous studies, our univariate analysis showed that advanced patient age ( $>50$ years), longer AF duration, a fine AF pattern (wave length $<1 \mathrm{~mm}$ ), and increased cardiothoracic ratio $(>60 \%)$ were associated with late $\mathrm{AF}$ recurrence. ${ }^{9,11-13}$

The size of the LA has been associated with failure of the maze procedure, because the ability of the LA to fibrillate is determined by the relationship between the effective refractory period of the atrial myocardium and the atrial area available for the macro-re-entrant circuit. Because atrial size reduction was shown to predict the long-term success of sinus conversion, ${ }^{14}$ we routinely performed LA reduction plasty in all patients with a giant LA. That the LA size was reduced in all patients, regardless of their preoperative LA size, might explain our finding that a cardiothoracic ratio $>60 \%$ was not a significant predictor of maze procedure failure.

TABLE 2. Univariate and multivariate risk factor analyses for recurrence of atrial fibrillation

\begin{tabular}{|c|c|c|c|c|}
\hline \multirow[b]{2}{*}{ Factors } & \multicolumn{2}{|c|}{ Univariate } & \multicolumn{2}{|c|}{ Multivariate } \\
\hline & $P$ value & HR & $95 \%$ CI & $P$ value \\
\hline \multicolumn{5}{|l|}{ Preoperative } \\
\hline Age $(>50 \mathrm{y})$ & $.001 *$ & 2.13 & $1.10-4.00$ & $.020 *$ \\
\hline $\mathrm{AF}$ duration $(>5 \mathrm{y})$ & $.013 *$ & 1.50 & $1.18-2.43$ & .712 \\
\hline Fine $(<1 \mathrm{~mm}) \mathrm{AF}$ wave pattern (vs coarse wave) & $.012 *$ & 1.66 & $0.88-3.09$ & .113 \\
\hline Persistent AF type (vs paroxysmal AF) & .749 & & & \\
\hline Left ventricular ejection fraction $(<50 \%)$ & .786 & & & \\
\hline Rheumatic mitral disease (vs degenerative) & .274 & & & \\
\hline Previous cardiac surgery & .285 & & & \\
\hline Cardiothoracic ratio $(>60 \%)$ & $.012 *$ & 1.69 & $0.85-2.82$ & .137 \\
\hline \multicolumn{5}{|l|}{ Operative } \\
\hline Mitral replacement (vs repair) & .386 & & & \\
\hline Cryoablation ablation (vs microwave ablation) & .159 & 0.62 & $0.31-1.21$ & .163 \\
\hline Concomitant coronary bypassing & .430 & & & \\
\hline Concomitant aortic valve surgery & .260 & & & \\
\hline Concomitant coronary + aortic valve surgery & .475 & & & \\
\hline Surgical period (1999-2002 vs 2003-2006) & .861 & & & \\
\hline
\end{tabular}




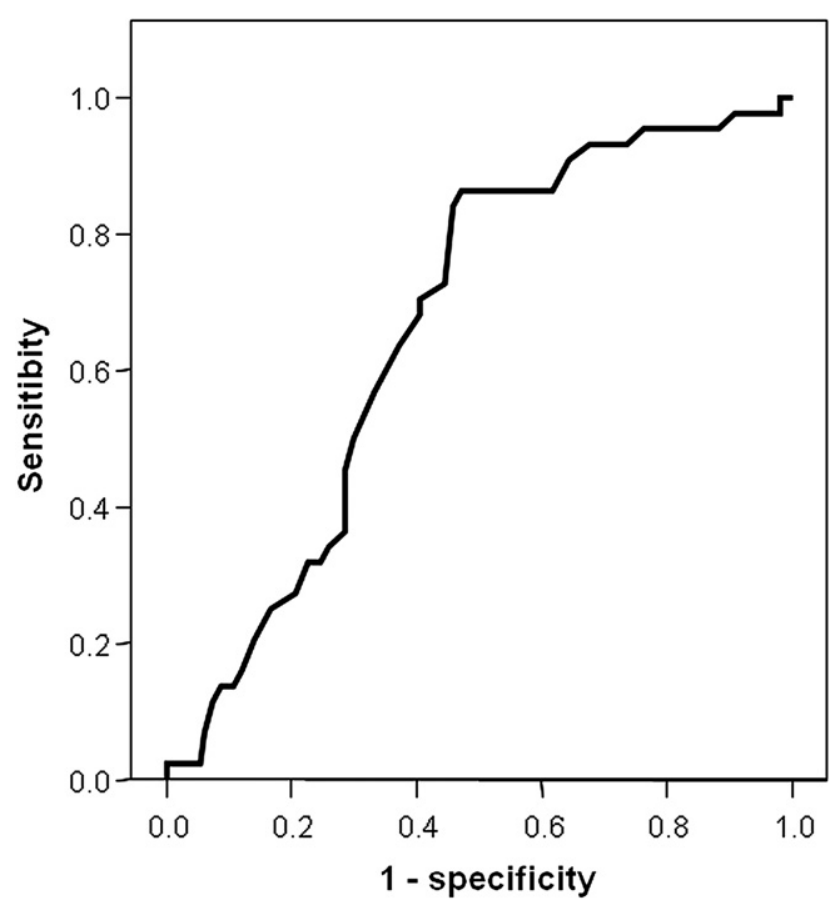

FIGURE 2. Receiver operating characteristic curve assessing cutoff value of age related to atrial fibrillation recurrence.

In assessing the differences in recurrence rates among patients with a reduced LA, we also determined the contribution of AF duration to the restoration of sinus rhythm. A prolonged AF duration ( $>6$ months), which has been particularly associated with mitral valve disease, has also been associated with a poor conversion rate to sinus rhythm after the maze procedure. ${ }^{15}$ Exposure to prolonged AF might induce pathologic changes to the atrial interstitial tissue associated with distension. However, a high rate of conversion to sinus rhythm was observed in patients with combined mitral disease, despite a long AF duration, ${ }^{10}$ and in patients with long-standing preoperative AF. ${ }^{16}$ Our findings were in good agreement with these previous results, in that the AF recurrence rate did not correlate with the AF duration.

We, therefore, focused on patient age, which could be considered a marker of AF duration and the aging process in the atrial tissue. Atrial interstitial fibrosis has been shown to increase with age and has been observed in patients with AF. ${ }^{17}$ Using a receiver operating characteristic curve, we found that patient age between 46.5 and 47.5 years gave optimal sensitivity and specificity for AF recurrence. We, therefore, divided our patients into 2 groups, those 50 years old and younger and those older than 50 years, and found a statistically significant between-group difference in $\mathrm{AF}$ recurrence $(P=.001)$. Multivariate analysis showed that age older than 50 years was the only risk factor for failure of the maze procedure in patients with a giant LA. To date, however, clear pathologic differences in atrial tissue, which might induce ectopic conduction signals, have not been observed in young or old patients with giant LA tissue. Fibrosis and degenerative changes have been correlated with decreased expression of connexin $43,{ }^{18,19}$ further suggesting that age might be a strong predictor of sinus conversion after the maze procedure.

The present study had several limitations, including its retrospective design and the relatively small number of patients. We could not determine why age was the only factor associated with AF recurrence. This suggests the need to assess the outcomes in old and young patients with a normal LA and to examine giant LA histologic specimens in both groups of patients.

\section{CONCLUSIONS}

Although a giant LA has been found to be predictive of poor outcomes after the maze procedure, we found that young patients had more favorable rhythm outcomes than older patients, as evidenced by the lower rate of AF recurrence. These findings indicate that a more aggressive surgical approach to eliminate AF might be warranted in young patients, even those with a giant LA.

\section{References}

1. Cox JL, Schuessler RB, D'Agostino HJ Jr, Stone CM, Chang BC, Cain ME, et al. The surgical treatment of atrial fibrillation: III. Development of a definitive surgical procedure. J Thorac Cardiovasc Surg. 1991;101:569-83.

2. Prasad SM, Maniar HS, Camillo CJ, Schuessler RB, Boineau JP, Sundt TM, et al. The Cox maze III procedure for atrial fibrillation: Long-term efficacy in patients undergoing lone versus concomitant procedures. J Thorac Cardiovasc Surg. 2003;126:1822-7.

3. Kamata J, Kawazoe K, Izumoto H, Kitahara H, Shiina Y, Sato Y, et al. Predictors of sinus rhythm restoration after Cox maze procedure concomitant with other cardiac operations. Ann Thorac Surg. 1997;64:394-8.

4. Kobayashi J, Kosakai Y, Nakano K, Sasako Y, Eishi K, Yamamoto F. Improved success rate of the maze procedure in mitral valve disease by new criteria for patients' selection. Eur J Cardiothorac Surg. 1998;13:247-52.

5. Everett THIV, Olgin JE. Atrial fibrosis and the mechanisms of atrial fibrillation. Heart Rhythm. 2007;4(3 Suppl. 1):S24-7.

6. Lee JW, Choo SJ, Kim KI, Song JK, Kang DH, Song JM, et al. Atrial fibrillation surgery simplified with cryoablation to improve left atrial function. Ann Thorac Surg. 2001;72:1479-83

7. Beukema WP, Sie HT, Misier ARR, Delnoy PP, Wellens HJJ, Elvan A. Predictive factors of sustained sinus rhythm and recurrent atrial fibrillation after a radiofrequency modified maze procedure. Eur J Cardiothorac Surg. 2008; 34:771-5.

8. Je HG, Lee JW, Jung SH, Choo SJ, Song H, Yun SC, et al. Risk factors analysis on failure of maze procedure: Mid-term results. Eur J Cardiothorac Surg. 2009;36: 272-9.

9. Baek M-J, Na C-Y, Oh S-S, Lee C-H, Kim JH, Seo HJ, et al. Surgical treatment of chronic atrial fibrillation combined with rheumatic mitral valve disease: Effects of the cryo-maze procedure and predictors for late recurrence. Eur J Cardiothoracic Surg. 2006;30:728-36.

10. Romano MA, Bach DS, Pagani FD, Prager RL, Deeb GM, Bolling SF. Atrial reduction plasty Cox maze procedure: Extended indications for atrial fibrillation surgery. Ann Thorac Surg. 2004;77:1282-7.

11. Gaynor SL, Schuessler RB, Bailey MS, Ishii Y, Boineau JP, Gleva MJ, et al. Surgical treatment of atrial fibrillation: Predictors of late recurrence. J Thorac Cardiovasc Surg. 2005;129:104-11.

12. Itoh A, Kobayashi J, Bando K, Niwaya K, Tagusari O, Nakajima H, et al. The impact of mitral valve surgery combined with maze procedure. Eur J Cardiothorac Surg. 2006;29:1030-5. 
13. Szalay ZA, Skwara W, Klövekorn WP, Brunner-La Rocca HP, Knez I, Graves K, et al. Predictors of failure to cure atrial fibrillation with the mini-maze operation. J Cardiac Surg. 2004;19:1-6.

14. Chen MC, Chang JP, Guo GB, Chang HW. Atrial size reduction as a predictor of the success of radiofrequency maze procedure for chronic atrial fibrillation in patients undergoing concomitant valvular surgery. J Cardiovasc Electrophysiol. 2001; 12:867-74.

15. Chua YL, Schaff HV, Orszulak TA, Morris JJ. Outcome of mitral valve repair in patients with preoperative atrial fibrillation: Should the maze procedure be combined with mitral valvuloplasty? J Thorac Cardiovasc Surg. 1994;107: 408-15.
16. Williams MR, Stewart JR, Bolling SF, Freeman S, Anderson JT, Argenziano M et al. Surgical treatment of atrial fibrillation using radiofrequency energy. Ann Thorac Surg. 2001;71:1939-44.

17. Frustaci A, Chimenti C, Bellocci F, Morgante E, Russo MA, Maseri A. Histological substrate of atrial biopsies in patients with lone atrial fibrillation. Circulation. 1997;96:1180-4

18. Kostin S, Klein G, Szalay Z, Hein S, Bauer EP, Schaper J. Structural correlate of atrial fibrillation in human patients. Cardiovasc Res. 2002;54:361-79.

19. Luo MH, Li YS, Yang KP. Fibrosis of collagen I and remodeling of connexin 43 in atrial myocardium of patients with atrial fibrillation. Cardiology. 2006;107: 248-53. 